Research Article

\title{
Multiobjective Optimization for Pavement Network Maintenance and Rehabilitation Programming: A Case Study in Shanghai, China
}

\author{
Yufeng Sun, ${ }^{1,2}$ Min Hu $\mathbb{D}^{1,2}$ Wenbo Zhou, ${ }^{3}$ and Wei $\mathrm{Xu}^{1,2}$ \\ ${ }^{1}$ SILC Business School, Shanghai University, Shanghai 201800, China \\ ${ }^{2}$ SHU-SUCG Research Centre for Building Industrialization, Shanghai University, Shanghai 200072, China \\ ${ }^{3}$ Shanghai Tunnel Engineering Co., Ltd., Shanghai 200032, China \\ Correspondence should be addressed to Min Hu; minahu@163.com
}

Received 9 January 2020; Accepted 19 May 2020; Published 13 July 2020

Academic Editor: Dimitris Mourtzis

Copyright (c) 2020 Yufeng Sun et al. This is an open access article distributed under the Creative Commons Attribution License, which permits unrestricted use, distribution, and reproduction in any medium, provided the original work is properly cited.

This study investigates the pavement network maintenance and rehabilitation (M\&R) programming problem, over a certain planning horizon and in the context of limited funding. We designed an integer programming model to fulfill three purposes, namely, optimize the road conditions, minimize user disturbance costs, and minimize agency costs. We present a case study in which this model is applied to the pavement network of Shanghai. We investigate the results through the use of five M\&R strategies, to identify the Pareto-optimal trade-offs inherent in developing pavement network M\&R planning. The results demonstrate that there is a positive relationship between PCI improvement and user disturbance costs and between PCI improvement and agency costs. Additionally, we conduct a comparative analysis between agency and government-oriented strategies to evaluate the effectiveness and equity consideration. The findings suggest that the government-oriented strategy improves the pavement condition effectively with low user disturbance costs, and the agency-oriented strategy accounts for more equity consideration. Finally, we formulate an extension model that considers multiple road types, for application to pavement network M\&R programming. The results show that light rehabilitation and preventive maintenance are the most frequently implemented treatments on arterial roads and secondary trunk roads from the case network implementation. This study helps decision-makers identify the trade-offs made when developing a pavement network M\&R program.

\section{Introduction}

With the rapid development of Shanghai's pavement networks, by the end of 2016, the total length of the city's roads exceeded $13,000 \mathrm{~km}$; this increase has necessarily resulted in a constant increase in tasks related to urban pavement maintenance and management. To improve the effectiveness and efficiency of pavement management strategies, it is necessary to evaluate scientifically the service level of pavement conditions and use such evaluations to develop an efficient road network maintenance management planning system.

Road maintenance decision-making often involves addressing multiple and conflicting objectives and factors, such as road condition performance, maintenance costs, and user disturbance impact. Because funds are limited, decision-makers need to undertake pavement maintenance treatments only on certain sections of the road network, and this parameter increases the challenge inherent in this work.

Therefore, this study looks to help decision-makers develop an optimal pavement network maintenance and rehabilitation (M\&R) program over a planning horizon. Multiobjective optimization techniques are often used to create optimal pavement network maintenance programs (i.e., in which pavement sections are maintained at an acceptable service-condition level while incurring low agency costs and not creating significant amounts of user disturbance, environment pollution, or traffic hazard). However, trading off maintenance strategies under such conflicting objectives is difficult, and for each strategy, it requires salient 
knowledge [1]. Therefore, in developing a pavement network $M \& R$ program, it is essential that decision-makers compare representative options and select appropriate strategies.

This paper presents a multiobjective pavement network maintenance optimization model that considers three conflicting objectives, namely, the maximization of pavement condition improvement, the minimization of user disturbance costs, and the minimization of agency costs over the planning horizon. The remainder of this paper is organized as follows. Section 2 reviews the related works, and Section 3 demonstrates the background of the problem. The model is formulated in Section 4, and in Section 5, a smallscale, 16-section pavement network within Shanghai is illustrated to validate the feasibility of the proposed model. In Section 6, we undertake a comprehensive analysis of the trade-offs that occur when undertaking pavement network M\&R planning. In Section 7, we formulate and apply an extension model to a large-scale, 121-section pavement network. Section 8 offers concluding remarks.

\section{Literature Review}

Pavement maintenance programming related studies have been a popular research topic. Friesz and Fernandez [2] were the first to determine optimal maintenance policies for transport facilities. Fwa et al. [3] proposed a mathematical programming for routine network-level maintenance activities. Chan et al. [4] presented a genetic algorithm based approach to allocate available resource in the pavement maintenance programming to maintain within a pavement network the highest possible level of serviceability. Chakroborty et al. [5] presented a 0-1 linear integer programming to comprehensively determine maintenance strategies for a network of road sections. Ahmed et al. [6] developed a novel particle swarm optimization algorithm to determine the optimal pavement M\&R scheduling while considering two objectives. Throughout the literature, the objective has been maintaining an efficient pavement management system while effectively allocating limited resources.

Contributing to the large scale of the road network and the limited M\&R funds, the optimization techniques have become very essential to the road network M\&R planning. Traditionally, researchers have used single-objective optimization techniques, given the complexity inherent in multiobjective analysis [7-10]. In these single-objective optimization problems, requirements that are not selected as objective functions are imposed as constraints in model formulation; as a result, the solutions are suboptimal in comparison to those obtained through multiobjective optimization $[11,12]$.

Additionally, M\&R planning that considers only a single objective will tend to ignore other important objectives, making it difficult to undertake comprehensive analysis. Therefore, in recent decades, several researchers have conducted optimization analysis that involves multiple objectives. For example, Fwa et al. [11] developed a genetic-algorithmbased procedure for solving multiobjective network-level pavement maintenance programming problems. To establish a biobjective pavement M\&R scheduling program, Gao et al. [13] investigated and subsequently demonstrated a parametric method, using a real-world case in Dallas, TX. Meneses and Ferreira [12] presented a multiobjective decision-aid tool that incorporates maintenance costs and user costs, and they use the case study of Portugal's pavement management. Chen et al. [1] proposed a dichotomic approach to maximize the PV benefit (and minimize the PV cost) under annual budgetary and condition requirements. France-Mensah and O'Brien [14] developed a sustainable pavement management plan that incorporates road conditions, user costs, and environmental considerations.

The aforementioned literature on multiobjective optimization for pavement network maintenance tends to focus on $\mathrm{M} \& \mathrm{R}$ planning for pavement networks, but principally from the perspective of a department of transportation. In practice, however, other stakeholders are also involved in the decision-making process. For example, government requirements vis-à-vis road conditions and budgetary control influence road agencies' M\&R planning; this means there exists a conflict between the government and the road agency. To the best of our knowledge, a limited body of literature proposes models that consider the conflict of various stakeholders in the decision-making process. He and Sun [15] presented four optimal decision models in M\&R planning, but they do not elucidate the differences among the optimal solutions of various stakeholders. This study contributes to the literature by explicitly modeling $M \& R$ decisions from the separate perspectives of the government and the agency, followed by a detailed comparative analysis of various "optimal" solutions.

This paper also relates to the literature on "equity" consideration in M\&R programming, which considers how $M \& R$ funds should be fairly distributed since social equity is the most overlooked element of the decision-making process [16]. In recent studies, some researchers have incorporated and evaluated social equity in M\&R programming process, since effectiveness is not the sole objective $[14,17]$. However, when developing M\&R planning, most decision-makers do not have a formal set of evaluation metrics to assess social equity. France-Mensah et al. [18] provided both a quantitative approach and evaluation metrics to support the integration of social equity into highway $M \& R$ programming decision-making. However, the proposed models are limited, in that they put forward single-objective optimization programming that does not consider other factors (e.g., economic prosperity).

This study makes a threefold contribution to the literature. First, as it considers three conflicting objectives, it uses multiobjective optimization techniques to create solutions at different scales of pavement network M\&R planning. Second, it conducts a detailed comparative analysis of various "optimal" solutions, from the perspective of various stakeholders involved in M\&R programming development. Third, as only a few studies integrate social equity factors into pavement network M\&R planning, this study contributes to the literature by explicitly evaluating and comparing the equity of the outcomes derived from various solutions. 


\section{Problem Description}

The problem addressed in this study relates to the allocation of limited funds to pavement network M\&R programming over a certain planning horizon. Three stakeholders are involved in developing the pavement maintenance program, namely, the government, the agency, and the users. Each decision-maker needs to consider a variety of objectives and factors, and so when creating a maintenance plan for a pavement network in a certain planning period and with limited funding, trade-offs are inevitable. For example, the execution of too many heavy maintenance treatments will effectively improve the road condition, but it will also increase the user disturbance costs. More importantly, funding limitations mean that only a few road sections can be considered for heavy maintenance. The execution of a lowercost maintenance treatment may reduce both user disturbance costs and agency costs, but it will also lead to poor road conditions-conditions that are perhaps even lower than those dictated by the government-and thus reduce road safety. Therefore, for decision-makers, it is especially important to decide upon a pavement network M\&R plan under limited budgets, while considering conflicting objectives that involve a variety of maintenance treatments. This constitutes a multiobjective optimization problem.

\section{Model Formulation}

In this section, we formulate an integer programming model for the problem. The model is formulated for a pavement network of $I$ pavement with $J$ maintenance treatments over the $T$ planning periods. The model is a modified version of that of Wang et al. [19], which we adapt to suit the background of the problem. We consider three objective functions followed by a set of constraints that reflect the decisionmaking process. Since common users are not directly involved in the pavement network M\&R planning, we formulate separate models for the agency and the government.

\subsection{Notions}

$T$ : Periods in the planning horizon

I: Pavement sections

$J$ : Available pavement improvement treatments

$l_{i}$ : The length of section $i$

$w_{i}$ : The width of section $i$

$a_{i}$ : The annual average daily traffic of a pavement section $i$

$e_{i j}$ : The unit effectiveness of alternative $j$ for section $i$

$c_{i j}$ : The unit cost of alternative $j$ for section $i$

$u_{i j}$ : The unit disturbance cost of alternative $j$ for section $i$

$b_{t}$ : The available budget in period $t$

$n_{i j}$ : The number of times alternative $j$ can be used on pavement section $i$ in the planning period $m$ : Pavement condition score below which a section must be repaired

$g$ : The maximum pavement condition score allowed $s_{i t}$ : Road condition score for section $i$ in period $t$ with initial condition score $s_{i 0}$

$x_{i j t}: 1$ or $0 ; 1$ stands for alternative $j$ for section $i$ in period $t, 0$ otherwise

$d$ : Discount rate

$t^{*}$ : The year when the M\&R is applied

$\alpha_{i}, \beta_{i}$ : The parameters of deterioration rate, which can be determined by the historical data.

4.2. Objective Functions. Our model considers three main objectives, namely, pavement condition improvements, users disturbance cost, and agency costs. These objective functions are formulated in the following equations, respectively:

$$
\begin{aligned}
& \text { Maximize } Z_{1}=\sum_{t=1}^{T} \sum_{i=1}^{I} \sum_{j=1}^{J} e_{i j} l_{i} w_{i} x_{i j t}, \\
& \text { Minimize } Z_{2}=\sum_{t=1}^{T} \sum_{i=1}^{I} \sum_{j=1}^{J} \frac{1}{(1+d)^{t}} u_{i j} l_{i} a_{i} x_{i j t}, \\
& \text { Minimize } Z_{3}=\sum_{t=1}^{T} \sum_{i=1}^{I} \sum_{j=1}^{J} \frac{1}{(1+d)^{t}} c_{i j} l_{i} w_{i} x_{i j t} .
\end{aligned}
$$

Objective 1 focuses on the total pavement network condition improvement over the planning periods. Objective 2 is formulated as the total user disturbance costs for the pavement network throughout the planning horizon. Objective 3 is the total agency costs of all maintenance treatments for the pavement network during the planning horizon.

4.2.1. Agency Objectives. In this problem, the agency faces a dilemma in determining a balance among three objectives (i.e., the pavement condition score, user disturbance costs, and agency costs). This means the agency needs to minimize the agency costs while meeting the requirement of pavement condition score and concurrently reducing to some extent the user disturbance costs. This constitutes a multiobjective optimization problem, and so the weightage factors $\left(w_{1}, w_{2}\right.$ and $\left.w_{3}\right)$ are introduced to help decision-makers identify each objective's importance. Given these conflicting objectives, we create a minimization of the normalized composite objective function $\left(Z_{4}\right)$, based on the study of France-Mensah and O'Brien [14]. Thus, the composite objective function $\left(Z_{4}\right)$ is formulated as per

$$
\text { Minimize } Z_{4}=w_{1}\left(\frac{-Z_{1}}{Z_{1}^{\max }}\right)+w_{2}\left(\frac{Z_{2}}{Z_{2}^{\min }}\right)+w_{3}\left(\frac{Z_{3}}{Z_{3}^{\min }}\right),
$$


where, $w_{1}, w_{2}$, and $w_{3}$ represent the weightage factors for objectives 1, 2, and 3 , respectively; $Z_{1}^{\max }$ stands for the maximum value for objective 1 assuming $w_{1}=1, w_{2}=0$, and $w_{3}=0 ; Z_{2}^{\min }$ stands for the minimum value for objective 2 assuming $w_{1}=0, w_{2}=1$, and $w_{3}=0 ; Z_{3}^{\text {min }}$ stands for the minimum value for objective 3 assuming $w_{1}=0, w_{2}=0$, and $w_{3}=1$.

4.2.2. Government Objectives. In this problem, the government's goal is to maximize the road condition score of pavement network while minimizing the user disturbance costs, subject to funding limitations over the planning horizon. Thus, the objective function $\left(Z_{5}\right)$ is as seen in

$$
\text { Minimize } Z_{5}=w_{1}\left(\frac{-Z_{1}}{Z_{1}^{\max }}\right)+w_{2}\left(\frac{Z_{2}}{Z_{2}^{\min }}\right)
$$

where $w_{1}$ and $w_{2}$ represent the weightage factors for objectives 1 and 2, respectively; $Z_{1}^{\max }$ stands for the maximum value for objective 1 assuming $w_{1}=1$ and $w_{2}=0 ; Z_{2}^{\min }$ stands for the minimum value for objective 2 assuming $w_{1}=$ 0 and $w_{2}=1$.

\subsection{Model Constraints}

$$
\begin{aligned}
& \text { s.t. } \sum_{i=1}^{I} \sum_{j=1}^{J} \frac{1}{(1+d)^{t}} c_{i j} l_{i} w_{i} x_{i j t} \leq b_{t}, \quad \forall t \\
& \sum_{t=1}^{T} x_{i j t} \leq n_{i j}, \quad \forall i, j, \\
& \sum_{j=1}^{J} x_{i j t}=1, \quad \forall i, t, \\
& s_{i t}=s_{i 0} e^{\alpha_{i} t+\beta_{i}}+\sum_{t^{*}=1}^{T} \sum_{j=1}^{J} x_{i j t} e_{i j} \cdot e^{\alpha_{i}\left(t-t^{*}\right)+\beta_{i}}, \quad \forall i, t, t^{*} \leq t \\
& s_{i t} \geq m, \quad \forall t, \\
& s_{i t} \leq g, \quad \forall t, \\
& x_{i j t} \in\{0,1\}, \quad \forall i, j, t .
\end{aligned}
$$

Constraints (6) ensure the total expenditure on maintenance treatments is within each year's budget. Constraints (7) ensure the frequency of maintenance treatments is limited. Constraints (8) indicate that only one maintenance action could be executed for each road section in each period. Constraints (9) show the road condition score is divided into two terms: the initial road condition score $s_{i 0}$ and the increased pavement maintenance effectiveness, where $e^{\alpha_{i}\left(t-t^{*}\right)+\beta_{i}}$ is the pavement condition transition process with the exponential deterioration rate which is based on He and Sun [15]. Constraints (10) and (11) are the minimum and maximum road condition score requirements, respectively. Constraints (12) assure integrality of the decision variable.

\section{Model Application and Experiments}

In this section, we apply the proposed models to a practical problem: developing a pavement network M\&R program in Shanghai, China, which features limited funding. In order to validate the proposed model's feasibility efficiently, it is appropriate to use a mathematical programming solver. Here, the integer programming model is solved by the branch-and-bound algorithm from the CPLEX Optimizer since it can produce the precise results efficiently. The optimization studio provides the NET API, and the solving process of the experiments is implemented based on that API. The mathematical model is coded in C\# language with Visual Studio 2015 on a personal computer (Inter Core i7, 3.4 GHz; memory, $8 \mathrm{G}$ ).

5.1. Experiment Background. The model was used with data pertaining to a subset pavement network in Shanghai, China. That network comprises 16 major arterial pavement sections with a total of over $100,000 \mathrm{~m}^{2}$ of maintenance area. We adopt a five-year planning time horizon for developing a pavement network M\&R program; this is practical and allows for a better evaluation of the trade-offs inherent in various maintenance treatments. Table 1 identifies the length, width, initial Pavement Condition Index (PCI), and annual average daily traffic (AADT) of each major arterial pavement section; these data are drawn from the 2015 pavement technical condition report for Shanghai [20].

5.2. Parameters and Assumption. The outcomes of $\mathrm{M} \& \mathrm{R}$ planning are often evaluated in terms of the road condition score improvement, which may be assessed by different overall condition indices such as the Pavement Condition Index (PCI) [21] or the Pavement Quality Index (PQI) [22]. In this study, PCI was used to measure the performance of road condition, which ranges from 0 to 100 (where 100 is perfect). Pavement should satisfy the minimum PCI requirement demanded by the government, and according to the Shanghai Road Bureau, the minimum PCI requirement for arterial roads within the city is 75 . When the PCI score is below 75, M\&R actions are initiated. However, different $M \& R$ projects can engender different levels of maintenance effectiveness and at different costs. Thus, in this study, we consider five different levels of M\&R actions: doing nothing (DN), preventive maintenance (PM), light rehabilitation (LR), medium rehabilitation (MR), and heavy rehabilitation (HR). Table 2 provides descriptions of each of these M\&R treatments, along with their average unit cost, user disturbance unit cost, and PCI improvement. These data are drawn from previous studies $[14,19,20]$.

Other data used in this study include annual budget, the discount rate, and deterioration-rate parameters. We assume the annual budget for this problem to be 10 million yuan and the annual discount rate to be $5 \%$. Finally, in line with 
TABLE 1: Basic information of pavement segments.

\begin{tabular}{|c|c|c|c|c|c|c|}
\hline Segment & Starting & Ending & Length (m) & Width (m) & PCI & AADT \\
\hline 1 & Qingchi Road & Jinzhong Road & 190.50 & 11.50 & 86.77 & 15000 \\
\hline 2 & Qingchi Road & Jinzhong Road & 190.50 & 27.20 & 86.77 & 15000 \\
\hline 3 & Wuzhong Road & Chengjia Bridge & 901.00 & 9.00 & 75.00 & 15000 \\
\hline 4 & Wande Road & Luoshan Road & 403.00 & 19.00 & 88.58 & 21000 \\
\hline 5 & Deping Road & Wande Road & 255.00 & 16.20 & 83.99 & 21000 \\
\hline 6 & Longju Road & Depin Road & 278.00 & 22.00 & 84.45 & 21000 \\
\hline 7 & Weifang Road & Pudian Road & 256.00 & 18.90 & 89.70 & 15000 \\
\hline 8 & Miaopu Road & Juye Road & 229.00 & 29.00 & 52.61 & 15000 \\
\hline 9 & Xiangyin Road & Jiamusi Road & 683.00 & 21.80 & 74.68 & 15000 \\
\hline 10 & Jiamusi Road & Songhuajiang Road & 364.00 & 23.70 & 75.00 & 15000 \\
\hline 11 & Jingjiang Road & Jiaotong Road & 101.00 & 6.00 & 72.26 & 15000 \\
\hline 12 & LiuYing Road & Taishan Branch Road & 82.50 & 17.70 & 75.00 & 15000 \\
\hline 13 & Huayin Road & LiuYing Road & 165.50 & 23.10 & 75.00 & 15000 \\
\hline 14 & Hutai Branch Road & Hutai Road & 352.00 & 31.40 & 71.50 & 15000 \\
\hline 15 & Xinhu Road & Baojia Bridge & 388.00 & 24.10 & 88.74 & 15000 \\
\hline 16 & Luochuan Road & Huayin Road & 257.50 & 23.10 & 75.00 & 15000 \\
\hline
\end{tabular}

TABLe 2: Description and key parameters of each M\&R treatment.

\begin{tabular}{|c|c|c|c|c|}
\hline $\begin{array}{l}\text { M\&R } \\
\text { treatment }\end{array}$ & Treatment description & $\begin{array}{l}\text { Average unit cost } \\
\quad\left(¥ / \mathrm{m}^{2}\right)\end{array}$ & $\begin{array}{l}\text { User disturbance unit cost ( } ¥ 10,000 / \\
\text { AADT } / \mathrm{km})\end{array}$ & $\begin{array}{c}\text { PCI } \\
\text { improvement }\end{array}$ \\
\hline $\mathrm{DN}$ & No M\&R projects applied & 0 & 0 & 0 \\
\hline PM & Chip seal; microsurfacing treatments & 20 & 1.34 & 3 \\
\hline LR & Thin overlays (2-3 in. thick) & 84 & 2.68 & 15 \\
\hline MR & Overlays ( $3-5$ in. thick) & 195 & 4.69 & 25 \\
\hline HR & $\begin{array}{l}\text { Reconstruction includes thick overlays } \\
\text { and milling }\end{array}$ & 350 & 6.70 & 40 \\
\hline
\end{tabular}

historical data, we assume the parameters of the deterioration rate $\left(\alpha_{i}, \beta_{i}\right)$ to be -0.05 and -0.02 , respectively.

\section{Results and Discussion}

In this section, we define five pavement treatment strategies in response to this problem, to identify the trade-offs made when developing a pavement network M\&R program; these strategies are discussed in terms of agency and government perspectives. The first three strategies are extreme options that focus on only one objective; from both the agency and government perspectives, the other two strategies are equalpriority strategies. Table 3 describes the five strategies.

6.1. Analysis of Results. In this study, we measure the performance of M\&R programs in terms of PCI improvement firstly. Figure 1 displays the average network condition scores for each strategy over the five-year planning horizon. The results show that strategy 1 seems to be the optimal pavement performance strategy: its average PCI in each year is greater than that of the other strategies (Figure 1). In order to illustrate the results of other objective functions, we normalized the results of each objective function into the same range. A min-max scaling method is adopted to scale the results to a given minimum and maximum value, usually between 0 and 1; the positive objective results (like PCI improvement) and negative objective results (like user disturbance costs and agency costs) are calculated by the following equations, respectively:

$$
y_{i j}=\frac{x_{i j}-\min _{1 \ll i \ll m} x_{i j}}{\max _{1 \ll i \ll m} x_{i j}-\min _{1 \ll i \ll m} x_{i j}}, \quad 1 \ll i \ll m, 1 \ll j \ll n,
$$

$$
y_{i j}=\frac{\max _{1 \ll i \ll m} x_{i j}-x_{i j}}{\max _{1 \ll i \ll m} x_{i j}-\min _{1 \ll i \ll m} x_{i j}}, \quad 1 \ll i \ll m, 1 \ll j \ll n .
$$

The comparison results of each strategy are shown by a radar map (Figure 2). Even though strategy 1 performs best in terms of PCI improvement, it incurs the highest user disturbance costs and agency costs. Strategy 2 controls the lowest user disturbance costs. Strategy 3 is the optimal agency costs strategy, but it also has the worst performance, given that it has the lowest number of M\&R projects. The other two equal-priority strategies (strategies 4 and 5) show moderate performance, as they represent compromises on the other objectives. Additionally, strategy 5 is more "effective" than strategy 4 in terms of total PCI improvement, even though the user disturbance costs of both strategies are very close.

6.2. Optimal Solution for Developing an M↔R Plan. In this section, we propose the optimal solution as determined during our experiment. As mentioned, strategies 1-3 involve extreme situations, while strategies 4 and 5 are equal-priority policies that consider multiple objectives and are representative of agency and government strategies, respectively. 
TABLe 3: The description and weightage set of the five strategies.

\begin{tabular}{lcc}
\hline Strategy number & Strategy objective(s) & Weightage ratios of each strategy \\
\hline 1 & Optimal pavement condition improvement & $w_{1}=1, w_{2}=0$, and $w_{3}=0$ \\
2 & Optimal user disturbance costs & $w_{1}=0, w_{2}=1$, and $w_{3}=0$ \\
3 & Optimal agency costs & $w_{1}=0, w_{2}=0$, and $w_{3}=1$ \\
4 & Equal priority (agency perspective) & $w_{1}=0.33, w_{2}=0.33$, and $w_{3}=0.33$ \\
5 & Equal priority (government perspective) & $w_{1}=0.5, w_{2}=0.5$, and $w_{3}=0$ \\
\hline
\end{tabular}

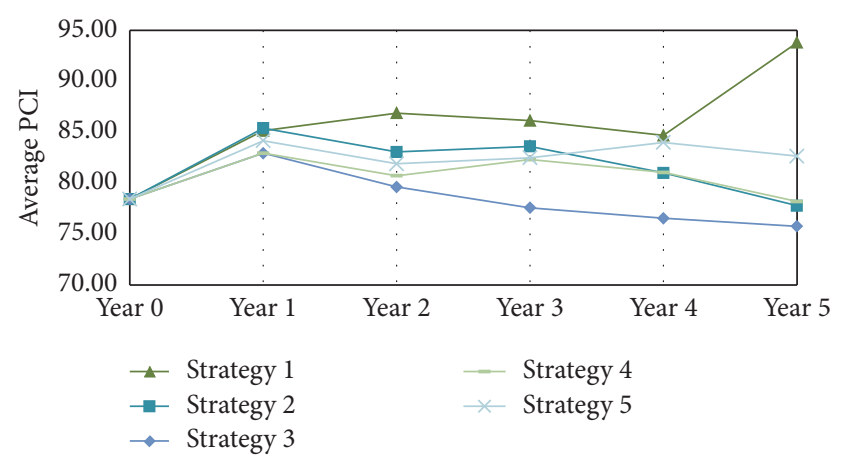

Figure 1: Average PCI of each strategy, in each study year.

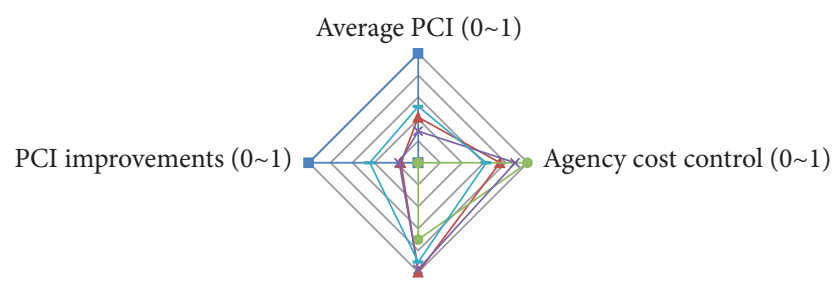

User disturbance cost control $(0 \sim 1)$

$\begin{array}{ll}\rightarrow \text { Strategy } 1 & \times \text { Strategy } 4 \\ \rightarrow \text { Strategy } 2 & - \\ \rightarrow \text { Strategy } 5\end{array}$

FIGURE 2: Results of each strategy throughout the planning horizon (16-segment experiment).

Here, we compare the results of these two latter strategies to illustrate $M \& R$ planning. The best $M \& R$ plans under the two strategies in this experiment are illustrated in Figure 3. As Figure 3 shows, both strategies 4 and 5, the HR are only applied to the eighth pavement in the first year. This happens because the initial PCI of the eighth pavement is only 52.61, which is far below the minimum PCI requirement. Additionally, for both strategies, LR accounts for most of the $M \& R$ projects across the entire planning horizon (Figure 4). However, strategy 4 considers more PM and less rehabilitation over the planning horizon than does strategy 5; this implies that strategy 4 constitutes a "friendly" pavement maintenance programming, because the overall impact of $\mathrm{M} \& \mathrm{R}$ on arterial pavement networks is relatively small.

6.3. Comparative Analysis between Agency and GovernmentOriented Strategies. In this section, we compare the results of the agency-oriented strategy (i.e., strategy 4) to those of the government-oriented strategy (i.e., strategy 5). In this case, strategy 5 is more effective than strategy 4 in terms of PCI improvement, even as the user costs of these strategies over the entire analysis period are nearly identical. Therefore, in this experiment, strategy 5 emerges as an "effective" strategy.

However, when developing an M\&R project plan for a pavement network, effectiveness is not the only standard to evaluate performance: recent studies $[23,24]$, for example, incorporate an equity factor into this problem. The common evaluation metrics in assessment of equity in M\&R projects are range in network condition score and standard deviation of road condition score [25]. In this study, we introduce two indicators to evaluate equity in the outcomes of various strategies. The two specific indicators are defined as the PCI range (the difference between the maximum and minimum PCI values) and the standard deviation (SD) of PCI of the pavement network for each planning year. Figure 5 graphically demonstrates the equity results of strategy 4 and strategy 5. From year 2 to year 4, both strategies have nearly the same level of disparity between the PCI of individual sections; however, in year 1 and year 5, the PCI range and standard deviation in the pavement network under strategy 4 are lower than those of strategy 5. This means that strategy 4 reduces the PCI level differential in the pavement network, especially in comparison to strategy 5 . Therefore, even though strategy 4 is inferior to strategy 5 in terms of PCI improvement performance (Figure 2), it does appear to be a more "socially equitable" strategy.

6.4. Pareto Solutions. To analyze the relationships among various objectives, we discuss Pareto-optimal solutions and offer two pairs of objectives, namely, PCI improvement versus total user disturbance costs and PCI improvement versus total agency costs. We adopt the weighted sum method to construct the Pareto-optimal frontier [26]. It is clear that the relationship between PCI improvement and user disturbance costs is positive (Figure 6); therefore, the greater the number of $M \& R$ treatments is conducted, the more the user disturbance costs will increase. Additionally, the relationship between PCI improvement and agency costs is positive (Figure 7); hence, a greater number of $M \& R$ activities will lead to a greater increase in agency costs. In addition, when the PCI improvement value exceeds 370,000, the user disturbance costs show an obvious acceleration trend; this in turn indicates that while undertaking a large number of MR or HR projects will improve road performance, doing so will also significantly increase user disturbance costs. Because the user disturbance costs depend on the AADT and the length of the pavement section, 


\begin{tabular}{|c|c|c|c|c|c|c|c|c|c|c|c|c|c|c|c|c|}
\hline \multirow{2}{*}{ Period } & \multicolumn{16}{|c|}{ Pavement segments } \\
\hline & 1 & 2 & 3 & 4 & 5 & 6 & 7 & 8 & 9 & 10 & 11 & 12 & 13 & 14 & 15 & 16 \\
\hline Year 1 & 1 & 1 & 3 & 1 & 1 & 1 & 1 & 5 & 3 & 3 & 3 & 3 & 3 & 3 & 1 & 3 \\
\hline Year 2 & 1 & 1 & 1 & 1 & 3 & 3 & 1 & 1 & 1 & 1 & 1 & 1 & 1 & 1 & 1 & 1 \\
\hline Year 3 & 3 & 3 & 1 & 3 & 1 & 1 & 1 & 1 & 1 & 1 & 3 & 1 & 1 & 3 & 3 & 1 \\
\hline Year 4 & 1 & 1 & 2 & 1 & 1 & 1 & 3 & 1 & 3 & 2 & 1 & 2 & 2 & 1 & 1 & 2 \\
\hline Year 5 & 1 & 1 & 2 & 1 & 1 & 1 & 1 & 2 & 1 & 2 & 1 & 2 & 2 & 1 & 1 & 2 \\
\hline
\end{tabular}

\begin{tabular}{|c|c|c|c|c|c|c|c|c|}
\hline DN & Do nothing & 1 \\
\hline PM & Preventive maintenance & 2 \\
\hline
\end{tabular} \begin{tabular}{|c|c|c|c|c|}
\hline LR & Light rehabilitation & 3 \\
\hline MR & Medium rehabilitation & 4 \\
\hline
\end{tabular}

(a)

\begin{tabular}{|c|c|c|c|c|c|c|c|c|c|c|c|c|c|c|c|c|}
\hline \multirow{2}{*}{ Period } & \multicolumn{16}{|c|}{ Pavement segments } \\
\hline & 1 & 2 & 3 & 4 & 5 & 6 & 7 & 8 & 9 & 10 & 11 & 12 & 13 & 14 & 15 & 16 \\
\hline Year 1 & 1 & 1 & 4 & 1 & 1 & 1 & 1 & 5 & 3 & 3 & 4 & 3 & 3 & 3 & 1 & 3 \\
\hline Year 2 & 1 & 1 & 1 & 1 & 3 & 3 & 1 & 1 & 1 & 1 & 1 & 1 & 1 & 1 & 1 & 1 \\
\hline Year 3 & 3 & 3 & 1 & 3 & 1 & 1 & 1 & 1 & 1 & 1 & 1 & 1 & 1 & 3 & 3 & 1 \\
\hline Year 4 & 1 & 1 & 1 & 1 & 1 & 1 & 3 & 1 & 3 & 3 & 1 & 3 & 3 & 1 & 1 & 3 \\
\hline Year 5 & 1 & 3 & 1 & 1 & 1 & 1 & 1 & 3 & 1 & 1 & 1 & 1 & 1 & 3 & 1 & 1 \\
\hline
\end{tabular}

\begin{tabular}{|l|c|c|c|c|c|}
\hline DN & Do nothing & 1 \\
\hline PM & Preventive maintenance & 2 \\
\hline
\end{tabular}$\quad$\begin{tabular}{|c|c|c|c|c|}
\hline LR & Light rehabilitation & 3 \\
\hline MR & Medium rehabilitation & 4 \\
\hline
\end{tabular}

(b)

FIgURE 3: Solutions of 16-segment experiment: (a) results of strategy 4; (b) results of strategy 5.

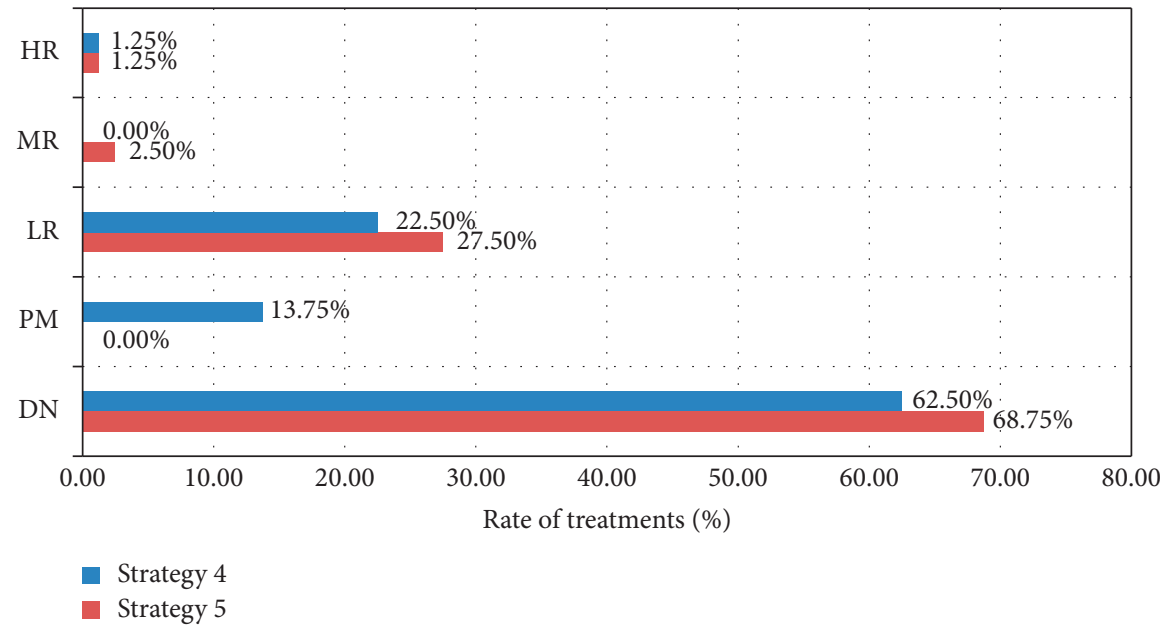

FIgURE 4: Types of M\&R projects in optimal maintenance programs.

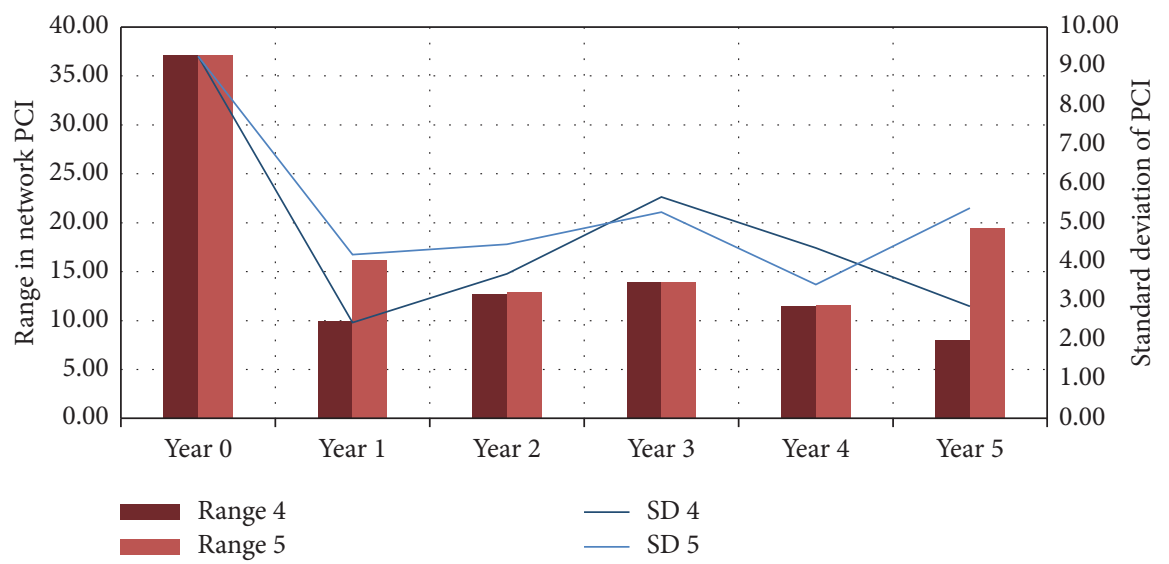

FIgURE 5: PCI range and standard deviation of PCI (16-segment experiment). 


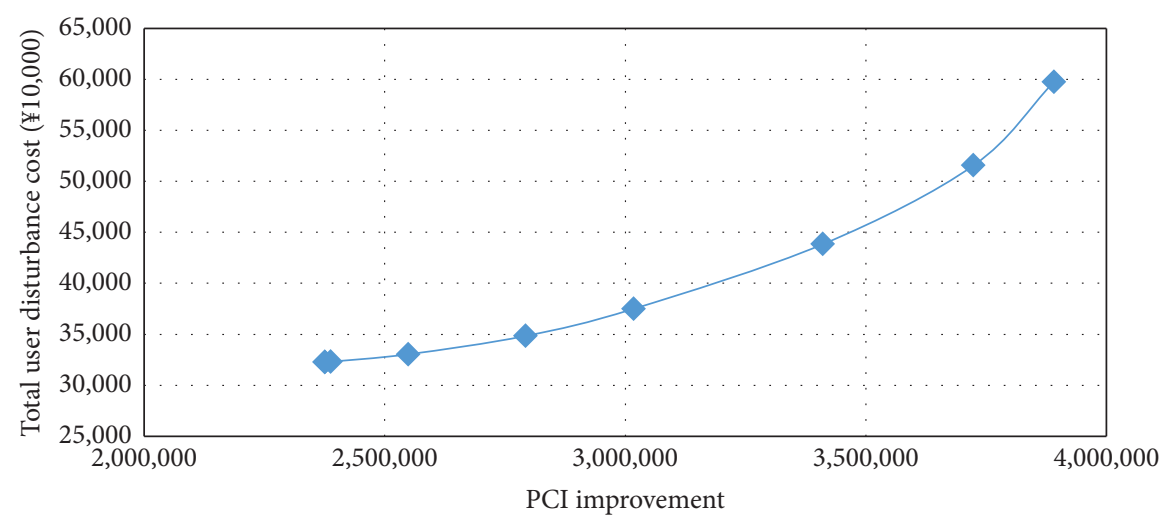

Figure 6: Pareto-optimal frontier for total user disturbance costs and total PCI improvement.

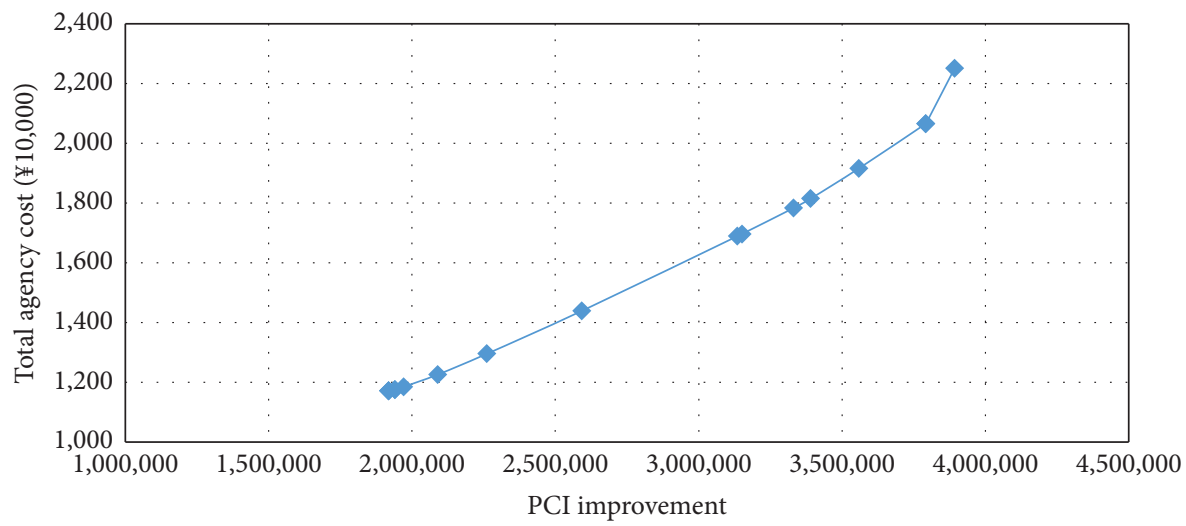

Figure 7: Pareto-optimal frontier for total agency costs and total PCI improvement.

decision-makers should take into account both factors when planning MR or HR treatments.

\section{Extension}

7.1. Model Extension. In this section, we examined a case where a pavement network includes different kinds of pavement, namely, arterial roads, secondary trunk roads, and branch roads. In reality, these different road types have different minimum road condition requirements. Thus, Constraints (10) can be modified as follows:

$$
\text { if } s_{i t}=k \text {, then } s_{i t} \geq m_{k}, k \in\{1,2,3 \ldots\}, \forall t,
$$

where $k$ is road type $k, s_{i t}$ is the road condition score for section $i$ in year $t$, and $m_{k}$ is the minimum road condition requirement of road type $k$.

7.2. A Large-Scale Experiment. We conducted a large-scale experiment involving 121 segments of road (over 430,000 m² of maintenance area in all), corresponding to an urban road network of Shanghai with 16 arterial roads, 44 secondary trunk roads, and 61 branch roads. For computation reasons, we adopted a five-year planning time horizon. The minimum requirement for the aforementioned road types is 75 , 70 , and 65 , respectively. The annual budget for this experiment was set at 30 million yuan. Other constraints, parameters, and assumptions are identical to those in the 16segment arterial road experiment.

7.3. Results and Discussion. As Figure 8 shows, the results of the 121-segment experiment indicate that strategies 4 and 5-which constitute the main treatments for arterial roads and secondary trunk roads-are LR and PM, respectively. For branch roads, the frequency of $M \& R$ projects throughout the planning horizon is lower than that of the other two road types in the pavement network.

In reviewing and comparing the results of different experimental scales (Figure 9), we see that the proportion of LR in the 16-segment experiment is larger than that in the 121-segment experiment; meanwhile, the proportion of PM in the 16-segment experiment is smaller than that in the 121segment experiment. These results stem from the facts that, in the 121-segment experiment, more LR is applied to arterial roads, and that while secondary trunk roads need no LR, they need more PM.

In addition, when we compare the optimal results of the various strategies, we see that, in either the small- or largescale experiments, strategy 4 has a higher proportion of PM than does strategy 5. However, the frequency of each of LR, $\mathrm{MR}$, and HR in strategy 4 is lower than that in strategy 5 , and this implies that strategy 4 is a more "friendly" strategy that employs more PM and less HR over the planning horizon. 


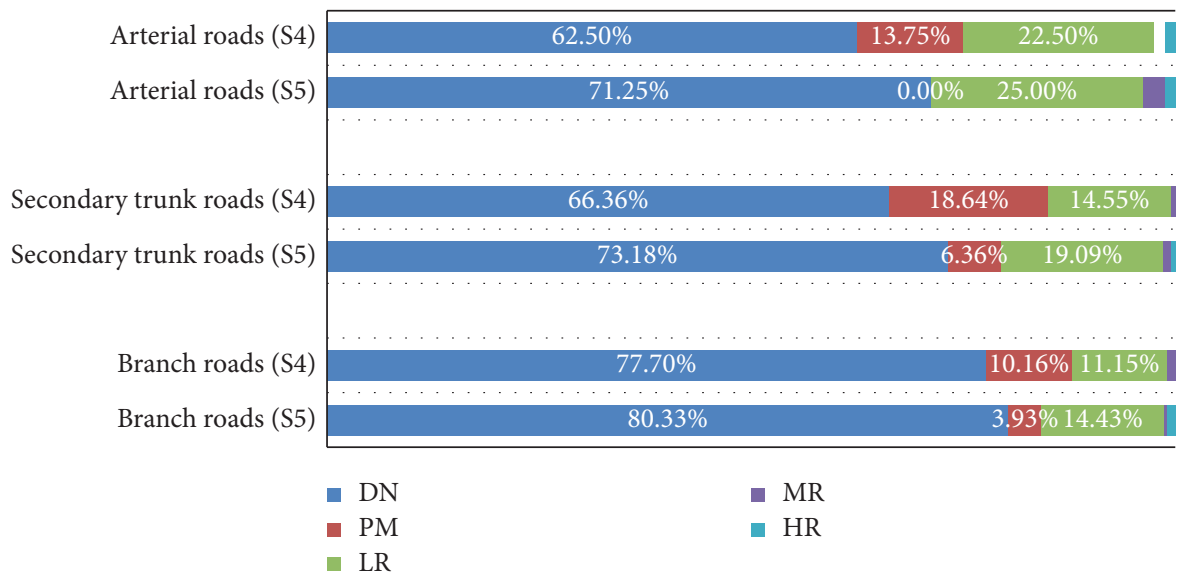

FIGURE 8: Types of M\&R projects in the 121-segment experiment (S4: strategy 4; S5: strategy 5).

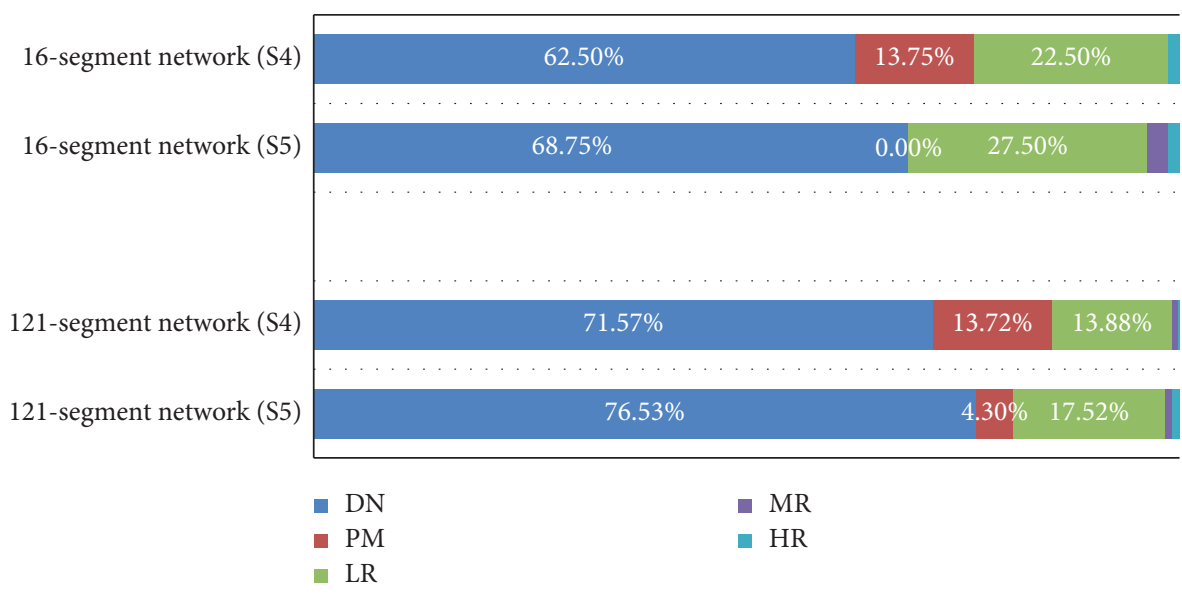

Figure 9: Comparison of different pavement network scales (S4: strategy 4; S5: strategy 5).

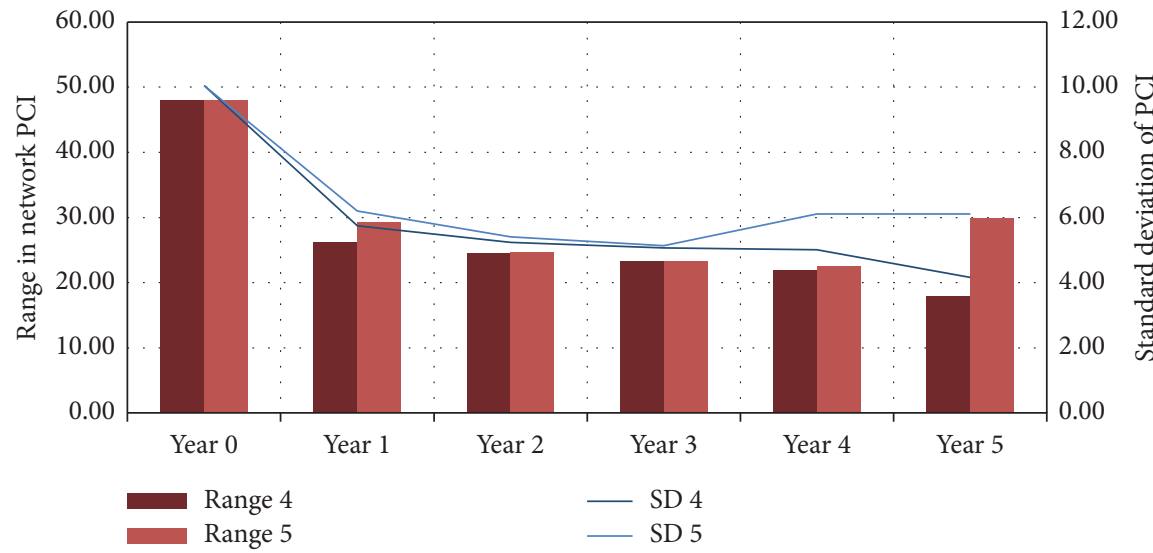

Figure 10: PCI range and standard deviation of PCI (121-segment experiment).

In the 121-segment experiment, the PCI range and standard deviation are used to evaluate equity in outcome. Figure 10 shows that, compared to strategy 5 , strategy 4 has a lower level of disparity between the PCI of individual sections in each year: especially in year 5, strategy 4 conspicuously reduces the PCI level differential in the pavement network. This result is consistent with that of the 16-segment experiment, which implies that strategy 4 is a more "equitable" strategy.

\section{Conclusion}

In this paper, we present a comprehensive means of analyzing the trade-offs inherent in developing pavement 
maintenance plans; our method is based on an integer programming model that is defined to consider three objective functions: the pavement condition improvement objective function, the user disturbance costs objective function, and the agency costs objective function. We apply the proposed model to the case of a pavement network in Shanghai over a five-year planning horizon. Based on the proposed model, we developed five strategies and compared their performance while considering conflicting objectives. Because different decision-makers are involved in such problems, we undertook comparative analysis between agency and government-oriented strategies. Additionally, we explored Pareto-optimal solutions to identify the relationship between two pairs of objectives. Finally, we formulated an extension model to fit a large-scale pavement network that features different kinds of roads.

Our results are as follows. First, the government-oriented strategy ensures effectiveness in road condition improvement and at relatively low user disturbance costs. However, compared to the agency-oriented strategy, the government's strategy is worse in terms of equity consideration: the agency strategy features a lower road condition differential throughout the pavement network over the planning horizon. The findings from this study provide useful information for decision-makers to choose flexible strategy to achieve specific goals. In addition, in a pavement network that features various road types, light rehabilitation and preventive maintenance are the most frequently implemented treatments on arterial roads and secondary trunk roads, respectively. For branch roads, overall $M \& R$ treatments are conducted less frequently over the planning horizon than for the two other road types. Furthermore, based on Pareto-optimal solution analysis, there exists a positive relationship between PCI improvement and user disturbance costs and between PCI improvement and agency costs. Finally, when PCI improvement exceeds a certain value threshold, the user disturbance costs show an obvious acceleration trend; this suggests that decisionmakers should take the user disturbance factor into consideration when they plan the execution of a large number of medium or heavy rehabilitation activities, especially for pavement sections that have a high average amount of daily traffic.

Future research can explore multiobjective optimization for pavement network M\&R programming while considering the social equity objective function. Additionally, it should be borne in mind that, for a large-scale section of pavement and a long planning horizon, it would be impossible to solve the model by using the CPLEX solver; therefore, one should consider the use of heuristic algorithms, to improve both computational efficiency and solution quality.

\section{Data Availability}

The data are drawn from the 2015 pavement technical condition report of Shanghai; please visit http://www. highway.sh.cn/ueditor/upload/1454467687986.pdf.

\section{Conflicts of Interest}

The authors declare that there are no conflicts of interest regarding the publication of this paper.

\section{Acknowledgments}

This work was supported by the Science and Technology Commission of Shanghai Municipality (no. 18DZ1201204).

\section{References}

[1] L. Chen, T. F. P. Henning, A. Raith, and A. Y. Shamseldin, "Multiobjective optimization for maintenance decision making in infrastructure asset management," Journal of Management in Engineering, vol. 31, no. 6, Article ID 04015015, 2015.

[2] T. L. Friesz and J. E. Fernandez, "A model of optimal transport maintenance with demand responsiveness," Transportation Research Part B: Methodological, vol. 13, no. 4, pp. 317-339, 1979.

[3] T. F. Fwa, K. C. Sinha, and J. D. N. Riverson, "Highway routine maintenance programming at network level," Journal of Transportation Engineering, vol. 114, no. 5, pp. 539-554, 1988.

[4] W. T. Chan, T. F. Fwa, and K. Zahidul Hoque, "Constraint handling methods in pavement maintenance programming," Transportation Research Part C: Emerging Technologies, vol. 9, no. 3, pp. 175-190, 2001.

[5] P. Chakroborty, A. Agarwal, and A. Das, "Comprehensive pavement maintenance strategies for road networks through optimal allocation of resources," Transportation Planning and Technology, vol. 35, no. 3, pp. 317-339, 2012.

[6] K. Ahmed, B. Al-Khateeb, and M. Mahmood, "Application of chaos discrete particle swarm optimization algorithm on pavement maintenance scheduling problem," Cluster Computing, vol. 22, no. S2, 2018.

[7] Y. Ouyang and S. Madanat, "Optimal scheduling of rehabilitation activities for multiple pavement facilities: exact and approximate solutions," Transportation Research Part A: Policy and Practice, vol. 38, no. 5, pp. 347-365, 2004.

[8] L. Gao, C. Xie, and Z. Zhang, "Network-level multi-objective optimal maintenance and rehabilitation scheduling," in Proceedings of the Transportation Research Board Meeting, Washington, DC, USA, January 2010.

[9] J. Yoo and A. Garcia-Diaz, "Cost-effective selection and multi-period scheduling of pavement maintenance and rehabilitation strategies," Engineering Optimization, vol. 40, no. 3, pp. 205-222, 2008.

[10] J. M. de la Garza, S. Akyildiz, D. R. Bish, and D. A. Krueger, "Network-level optimization of pavement maintenance renewal strategies," Advanced Engineering Informatics, vol. 25, no. 4, pp. 699-712, 2011.

[11] T. F. Fwa, W. T. Chan, and K. Z. Hoque, "Multiobjective optimization for pavement maintenance programming," Journal of Transportation Engineering, vol. 126, no. 5, pp. 367-374, 2000.

[12] S. Meneses and A. Ferreira, "Pavement maintenance programming considering two objectives: maintenance costs and user costs," International Journal of Pavement Engineering, vol. 14, no. 2, pp. 206-221, 2013.

[13] L. Gao, C. Xie, Z. Zhang, and S. T. Waller, "Network-level road pavement maintenance and rehabilitation scheduling for optimal performance improvement and budget utilization," 
Computer-Aided Civil and Infrastructure Engineering, vol. 27, no. 4, pp. 278-287, 2012.

[14] J. France-Mensah and W. J. O'Brien, "Developing a sustainable pavement management plan: tradeoffs in road condition, user costs, and greenhouse gas emissions," Journal of Management in Engineering, vol. 35, no. 3, Article ID 04019005, 2019.

[15] Z.-q. He and X.-1. Sun, "Discrete optimization models and methods for management systems of pavement maintenance and rehabilitation," Journal of Shanghai University (English Edition), vol. 14, no. 3, pp. 217-222, 2010.

[16] M. D. Hendricks, M. A. Meyer, N. G. Gharaibeh et al., "The development of a participatory assessment technique for infrastructure: neighborhood-level monitoring towards sustainable infrastructure systems," Sustainable Cities and Society, vol. 38, pp. 265-274, 2018.

[17] S. D. Boyles, "Equity and network-level maintenance scheduling," EURO Journal on Transportation and Logistics, vol. 4, no. 1, pp. 175-193, 2015.

[18] J. France-Mensah, C. Kothari, W. J. O'Brien, and J. Jiao, "Integrating social equity in highway maintenance and rehabilitation programming: a quantitative approach," Sustainable Cities and Society, vol. 48, Article ID 101526, 2019.

[19] F. Wang, Z. Zhang, and R. Machemehl, "Decision-making problem for managing pavement maintenance and rehabilitation projects," Transportation Research Record: Journal of the Transportation Research Board, vol. 1853, no. 1, pp. 21-28, 2003.

[20] http://www.highway.sh.cn/ueditor/upload/1454467687986. pdf.

[21] M. Y. Shahin, Pavement Management for Airports, Roads, and Parking Lots, Springer, New York, NY, USA, 2005.

[22] M. A. Karan, T. J. Christison, A. Cheetham, and G. Berdahl, "Development and implementation of Alberta's pavement information and needs system," Transportation Research Record, vol. 938, pp. 11-20, 1983.

[23] E. M. Ferguson, J. Duthie, A. Unnikrishnan, and S. Travis Waller, "Incorporating equity into the transit frequencysetting problem," Transportation Research, Part A (Policy and Practice), vol. 46, no. 1, pp. 190-199, 2012.

[24] S. Mishra, M. M. Golias, S. Sharma, and S. D. Boyles, "Optimal funding allocation strategies for safety improvements on urban intersections," Transportation Research Part A: Policy and Practice, vol. 75, pp. 113-133, 2015.

[25] J. France-Mensah and W. J. O’Brien, "Budget allocation models for pavement maintenance and rehabilitation: comparative case study," Journal of Management in Engineering, vol. 34, no. 2, 2018.

[26] C. Torres-Machi, E. Pellicer, V. Yepes, and A. Chamorro, "Towards a sustainable optimization of pavement maintenance programs under budgetary restrictions," Journal of Cleaner Production, vol. 148, pp. 90-102, 2017. 